\title{
Polarization errors associated with birefringent waveplates
}

\author{
Edward A. West \\ Matthew H. Smith \\ NASA/Marshall Space Flight Center \\ Space Sciences Laboratory \\ ES82 \\ Marshall Space Flight Center, Alabama \\ 35812 \\ E-mail: edward.west@msfc.nasa.gov
}

\begin{abstract}
Although zero-order quartz waveplates are widely used in instrumentation that needs good temperature and field-of-view characteristics, the residual errors associated with these devices can be very important in high-resolution polarimetry measurements. How the field-ofview characteristics are affected by retardation errors and the misalignment of optic axes in a double-crystal waveplate is discussed. The retardation measurements made on zero-order quartz and single-order "achromatic" waveplates and how the misalignment errors affect those measurements are discussed.
\end{abstract}

Subject terms: polarization analysis and measurement; waveplates; retardation; polarimetry.

Optical Engineering 34(6), 1574-1580 (June 1995).

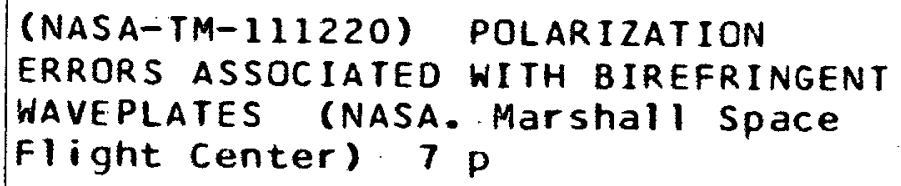

N96-17862

Unclas

$63 / 74 \quad 0098274$

\section{Introduction}

In almost any device that measures polarization, waveplates seem to be a necessary evil. They may be used as one of the active elements in a polarization analyzer (for example, a rotating quarter-wave plate ${ }^{1,2}$ ) or in the calibration of the polarization analyzer (for example, $\mathrm{KD}^{*} \mathrm{P}$ electro-optic crystal $^{3}$ ). As the accuracy of polarization measurements is pushed below $10^{-2}$, errors that might normally be neglected in waveplates become important, and determining the source of those errors so that models can be developed to study and eliminate them becomes a complicated task.

In instruments that measure polarized light very accurately, "known" polarizations are used as the input source, measurements are made with the polarimeter, and the Mueller matrix relating the input to the output is determined. This paper discusses the test equipment used and the data that were obtained on quarter-wave plates, which will be used in the development of a polarimeter for solar magnetic field measurements. Section 2 describes the test equipment and some of the problems that occurred in determining the ab-

Paper PAM-07 received Oct. 31, 1994; revised manuscript received Jan. 9, 1995; accepted for publication Jan. 26, 1995. This paper is a revision of a paper presented at the SPIE conference on Polarization Analysis and Measurement II, July 1994,

San Diego, CA. The paper presented there appears (unrefereed) in SPIE

Proceedings Vol. 2265.

(C) 1995 Society of Photo-Optical Instrumentation Engineers. 0091-3286/95/\$6.00. solute retardance. Section 3 describes the errors associated with multiple plate retarders and Sec. 4 presents the data that were obtained on the experimental vector magnetograph (EXVM) waveplates.

\section{Test Equipment}

Figure 1 shows the optical components that were used in the retardation measurements. One of the main problems in testing any polarization element is obtaining a perfect input polarizer and a perfect analyzer. In the visible spectrum, prism polarizers have the highest polarization resolution. To produce a "perfect" polarization source, two Glan-Thompson polarizers were aligned in parallel and were placed in front of the waveplate to be tested. The "perfect" analyzer was the EXVM polarimeter which consists of a Glan-Thompson polarizer and an HN32 Polaroid whose transmission axes were also aligned parallel. ${ }^{4}$

A multiline HeNe laser was used as the light source and a beam expander was added to the laser to produce a $13-\mathrm{mm}$ collimated beam. A shutter and an achromatic waveplate were placed between the linearly polarized laser and the calibration polarizer.

A photomultiplier (PM) tube was used as the detector. The PM tube was selected because of its sensitivity to low light levels. Because PM tubes are not known for their linearity and the input source (the multiline HeNe laser) was not stable, 


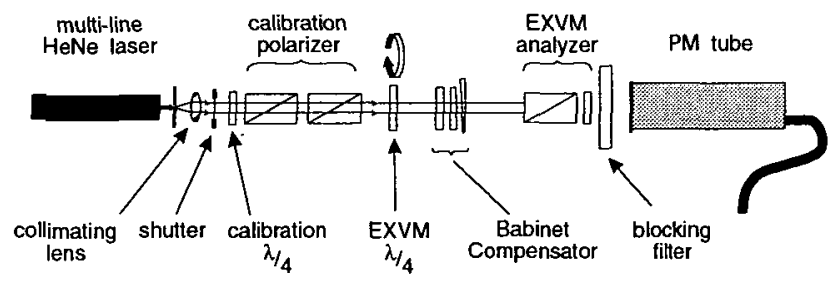

Fig. 1 Optical equipment used to test the EXVM quarter-wave plates.

a Soleil-Babinet compensator was used to measure the retardation of the EXVM waveplates. To minimize detector and source errors, all calibration points and retardation measurements were made at minimum signal levels and as rapidly as possible. With the detector and source errors minimized, the Soleil-Babinet compensator became the next critical error source.

In the following discussion, "optical axis" refers to the propagation direction of the light. "Optic axis" refers to the direction in a birefringent crystal in which there is no phase retardance for light propagating along that axis. For biaxial crystals, there are two "optic axes.' For uniaxial crystals, which are used in the construction waveplates, there is one optic axis direction and it is parallel to the extraordinary index of refraction $\left(n_{e}\right)$. Therefore, to change polarized light, the "optic axis" of a waveplate is perpendicular to the "optical axis" of the lens system.

The optical components of a Soleil-Babinet compensator are shown in Fig. 2. The compensator consists of three quartz plates, one plane parallel plate and two plates with a $2.5 \mathrm{deg}$ wedge. The optic axis (OA) of the parallel plate is perpendicular to the optic axes of the two wedge plates, and one of the wedge plates is allowed to move. The parallel plate has a fixed retardance, whereas the two wedges form a variable waveplate whose retardance is dependent on the total thickness of the two wedges. The parallel plate retarder is combined with the wedge retarder to minimize the variation of retardance over the field of view. As one would expect, the main error in the Soleil-Babinet compensator is the positioning of the moveable wedge. These include both rotational misalignment errors of the optic axes of the two wedges and translational errors (a thickness/optical path error, which translates into a retardation error). To minimize the translation and rotational errors in the compensator, the moveable waveplate was only allowed to move in one direction to minimize mechanical backlash in its positioning. Finally, the compensator was calibrated before and after every set of fast axis retardation measurements (at $45,135,225$, and $315 \mathrm{deg}$ ) made on the test waveplate. This was done to eliminate any systematic dc offsets in the retardation measurements.

\section{Modeling Retarder Errors}

There are some errors that depend on the quality control in the construction phase of the retarder that are difficult to model and are neglected here. One error that is neglected is the crystalline structure of the birefringent material used to produce the waveplate. This error is dependent on the experience of the optician and the availability of high-quality crystals. Other errors are related to the manufacturing process such as roll-off effects at the edge and stress-induced bire-

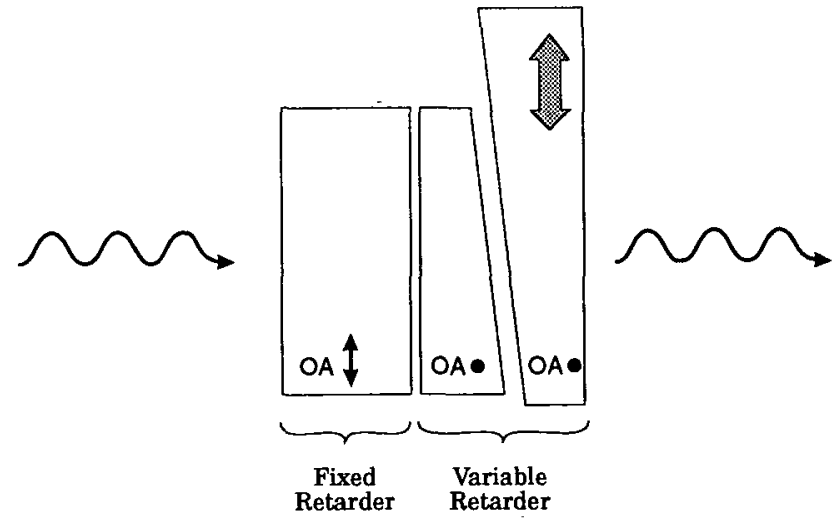

Fig. 2 Optical elements that make up a Soleil-Babinet compensator.

fringence. Because these errors are dependent on the experience of the company manufacturing the waveplates (and the user to detect them), they are neglected and the following assumptions are made: (1) there is no variation in the optical properties in the birefringent crystals used to produce the waveplates, (2) the aperture size of the retarder is much larger than the incident light so that edge effects can be neglected, and (3) the waveplates forming the retarder have parallel surfaces. The remaining errors are thickness, field-of-view errors, optic axis tilt errors, and fast axis misalignments. These errors are shown in Fig. 3. Before describing these errors, a short discussion on the two types of waveplates that were selected for use in the EXVM polarimeter is in order.

\subsection{Description of the EXVM Waveplates}

Figure 4 shows the structure of the zero-order and achromatic waveplates used in the EXVM magnetograph. The zero-order waveplates are standard quartz waveplates in which the optic axes (the extraordinary indices, $n_{y}$ and $n_{x}^{\prime}$, in Fig. 4) of the two quartz crystals are perpendicular to the propagation of the light (optical axis) and are separated by $90 \mathrm{deg}$. This orientation minimizes field-of-view errors and the retardation is simply related to the difference in the thickness of the two plates. Although the relationship of retardance to thickness is normally written as

$\delta=\frac{2 \pi\left(n_{e}-n_{o}\right)(\Delta d)}{\lambda}$,

we use a longer notation so that the errors to be discussed can be related to that equation. Therefore, the equation for a zero-order or achromatic retarder is written in the form

$\delta=\frac{2 \pi\left(n_{y}-n_{x}\right) d_{1}}{\lambda}+\frac{2 \pi\left(n_{y}^{\prime}-n_{x}^{\prime}\right) d_{2}}{\lambda}$,

or

$\delta=\delta_{1}+\delta_{2}$,

where $n_{x}$ is the ordinary index of refraction, $n_{y}$ is the extraordinary, and $\delta_{1}$ is the total retardance of the first waveplate (WP1). Similarly, $n_{y}^{\prime}$ is the ordinary, $n_{x}^{\prime}$ the the extraordinary, and $\delta_{2}$ the total retardance of the second waveplate (WP2). 


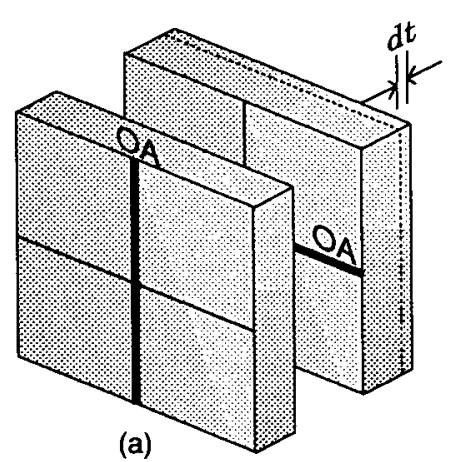

(a)

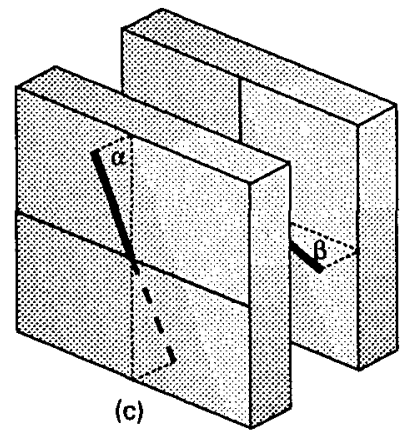

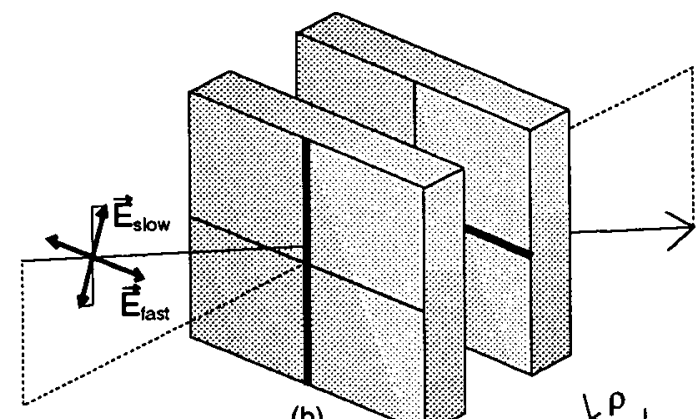

(b)

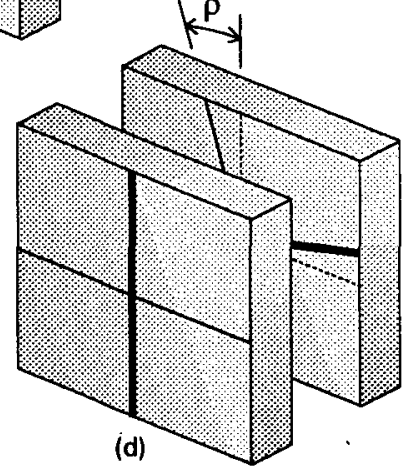

Fig. 3 Schematic of the various errors considered: (a) thickness error, (b) field-of-view errors, (c) optic axis tilt errors, and (d) fast axis misalignment.

The achromatic waveplates have the same optic axis alignment as the quartz waveplates, but one of the quartz plates is replaced with a magnesium fluoride $\left(\mathrm{MgF}_{2}\right)$ plate. Both quartz and $\mathrm{MgF}_{2}$ are positive uniaxial crystals $\left(n_{e}>n_{o}\right)$. Although termed an achromatic waveplate, the design goal was to achieve a quarter-wave retardance at 5250 and $6302 \AA$. Therefore, the thicknesses for the $\mathrm{MgF}_{2}$ and quartz plates were adjusted to meet those design specifications. Table 1 shows the optical properties that would be required to obtain perfect zero-order and achromatic waveplates. Figure 5 shows how the retardance of these waveplates varies with wavelength. These perfect waveplates are lused to demonstrate how the various errors affect the: performance of the EXVM retarders.

\subsection{Thickness Error}

The thickness error for a zero-order quartz waveplate is simply a dc offset, or shift in the retardation axis for the curves in Fig. 5. If the thickness of the first waveplate (WP1 in Table 1) is smaller (or larger) than the specification, the thickness of the second waveplate (WP2) is simply adjusted in the same way. The importance is in the difference in thickness, not the total thickness. For achromatic waveplates, however, the thickness tolerance is very critical if the achromat is to be quarter-wave at 5250 and $6302 \AA$. This results from the fact that there are only two independent equations ( $\delta$ at 5250 and $6302 \AA$ ) and two unknowns $\left(d_{1}\right.$ and $\left.d_{2}\right)$, for the achromatic waveplates. In the zero-order waveplates, there is one equation $(\delta$ at $5250 \AA)$ and one unknown $(\Delta d$, which is equal to $d_{1}-d_{2}$ ). Therefore, a thickness error in the EXVM achromatic waveplates is much more critical than a thickness error in the zero-order quartz waveplates. Figure 6 shows how the order of an achromatic waveplate affects its retardation versus wavelength properties. Varying the order $(m \times 360 \mathrm{deg}$,

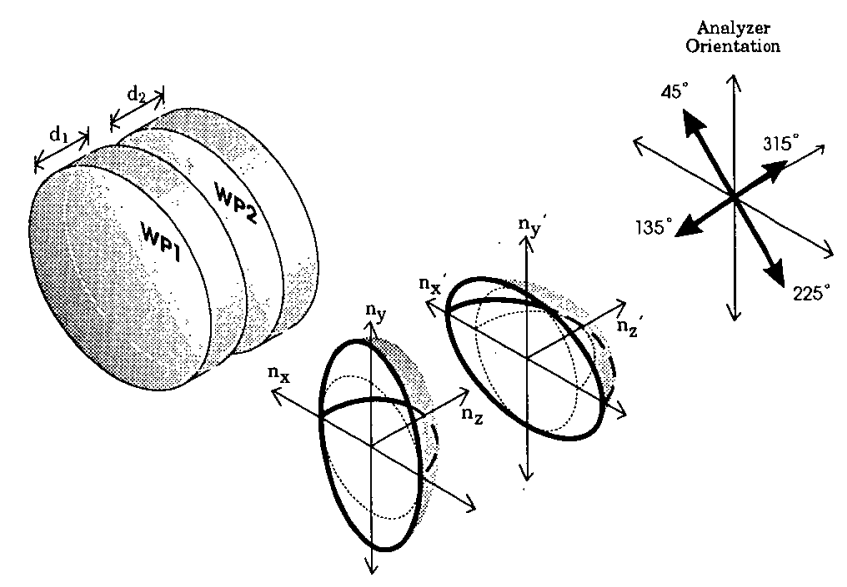

Fig. 4 Orientation of the optical properties in a double-waveplate retarder.

where $m$ is the order and $m=0,1,2, \ldots$ ) of the waveplate allows the total thickness to change while the thickness ratio remains constant.

\subsection{Field-of-View Errors}

Field-of-view errors exist in all birefringent elements. The birefringent property that enables creating a phase shift in a retarder is also the same property that limits its field of view. The field-of-view errors are related to the birefringence $\left(n_{e}-n_{o}\right)$ of the crystal, its thickness $\left(d_{1}, d_{2}\right)$, and the direction of the incident light. Assume that $\alpha+90$ deg is the angle that the light makes relative to the optic axis of the first crystal (WP1) and that $\beta+90$ deg is the angle relative to the optic axis of the second crystal (WP2) and that $\alpha$ and $\beta$ lie in orthogonal planes (see Fig. 7), then the maximum field-of- 
Table 1 Optical properties of ideal quarter-wave plates for use in the EXVM polarimeter. The values are at $5250.2 \AA$ and $22^{\circ} \mathrm{C}$.

\begin{tabular}{llllllll}
\hline Retarder & Plate \# & Material & $n_{x}$ & $n_{y}$ & $n_{x}-n_{y}$ & $\begin{array}{c}d \\
(\mathrm{~mm})\end{array}$ & $\begin{array}{c}\delta \\
(\text { degrees })\end{array}$ \\
\hline Zero-order & WP1 & quartz & 1.5473 & 1.5565 & $-9.207 \mathrm{e}-3$ & 2.5413 & -16044 \\
& WP2 & quartz & 1.5565 & 1.5473 & $+9.207 \mathrm{e}-3$ & 2.5270 & +15954 \\
\hline Achromatic & WP1 & quartz & 1.5473 & 1.5565 & $-9.207 \mathrm{e}-3$ & 1.612 & -10171 \\
& WP2 & MgF & 1.3909 & 1.3791 & $+1.186 \mathrm{e}-2$ & 1.305 & +10621 \\
\hline
\end{tabular}

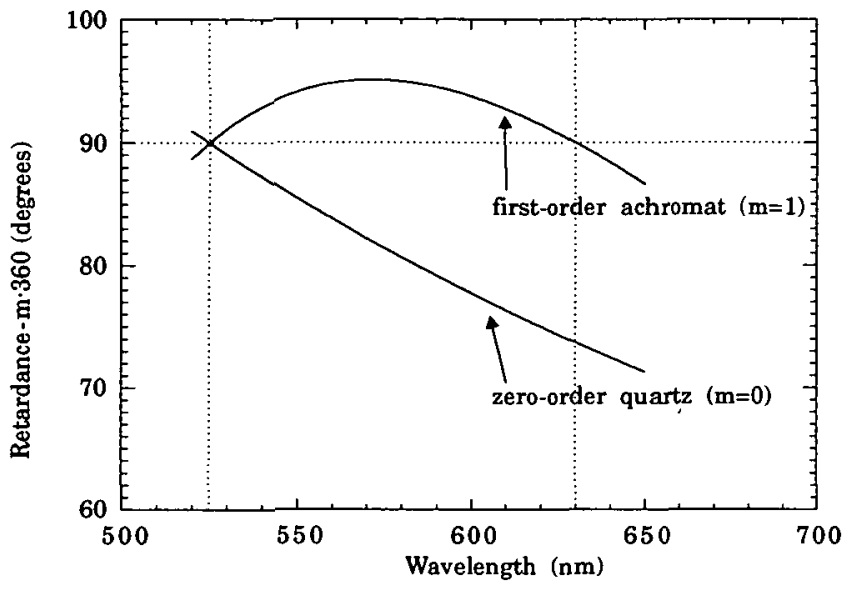

Fig. 5 Variation of retardance with wavelength for perfect zero-order and achromatic quarter-wave plates. For the achromatic waveplates the thicknesses were chosen so that the retarder would be a perfect $90 \mathrm{deg}(+360 \mathrm{deg})$ retarder at 5250 and $6302 \AA$.

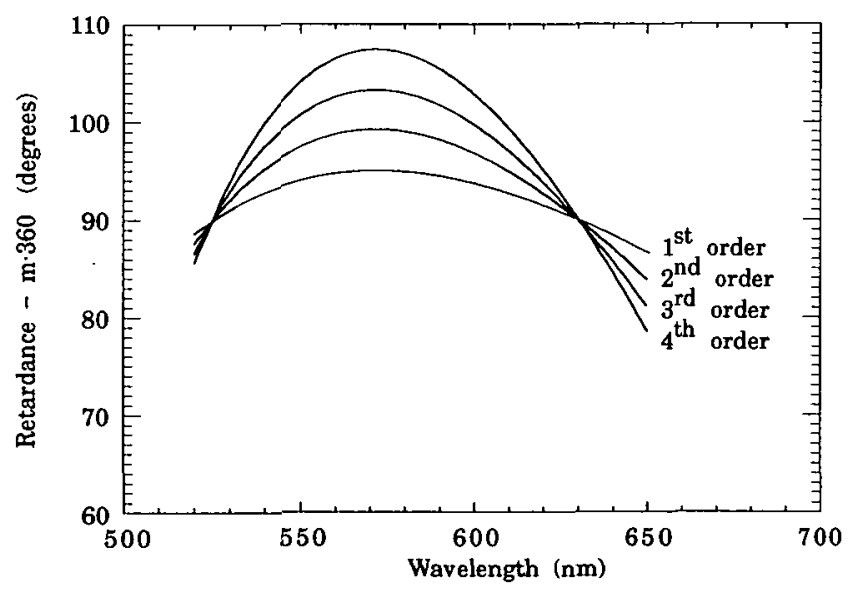

Fig. 6 Variation of retardance in a quartz/MgF 2 achromatic waveplate with the order $m(90 \mathrm{deg}+m \times 360 \mathrm{deg})$.

view errors will occur in those two planes. The optic axes in these two planes are normally referred to as the fast and slow axes of the retarder. When $|\alpha|=|\beta|$ there are no retardation errors (dotted lines in the retardance map shown in Fig. 7). The best way to understand field-of-view errors is to simply place a quarter-wave plate between crossed polarizers and rotate it until there is a maximum separation in the dark fringes that will be observed. The observed pattern will be

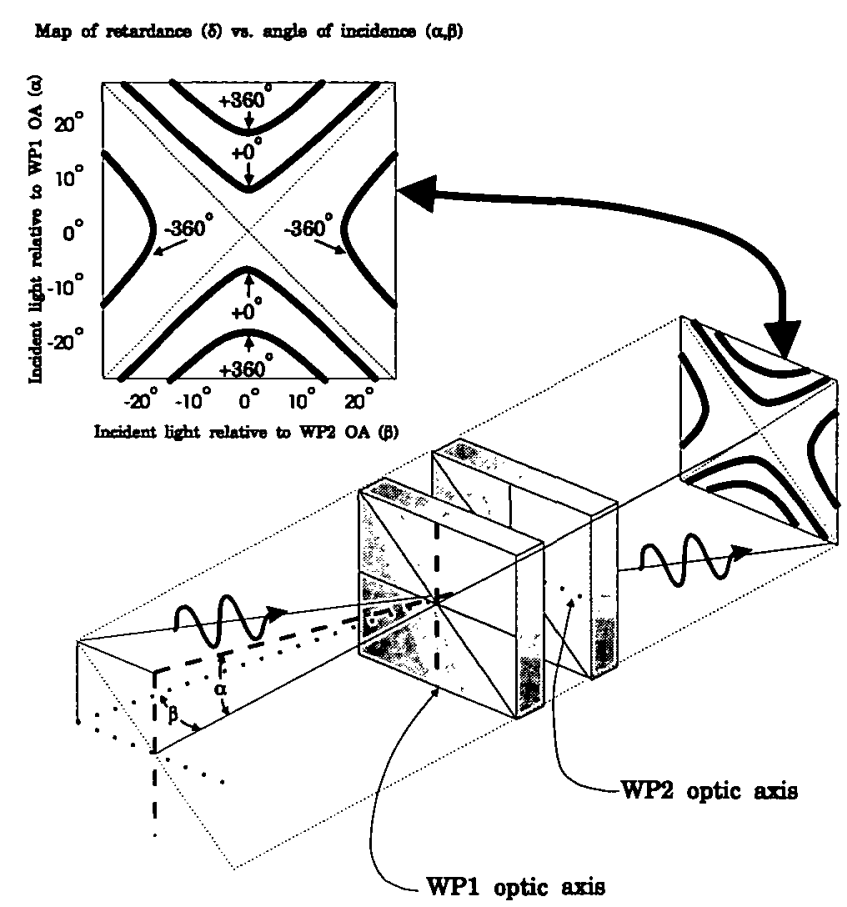

Fig. 7 Schematic showing how the retardation $\delta$ varies with the angle of incidence of the light relative to the optic axes of WP1 and WP2. The dotted lines in the retardance map represent the on-axis retardance [here assumed to be $\delta(0,0)=-90 \mathrm{deg}$ ]. The dark contours are retardance values relative to this on-axis retardance, for example $\delta(0 \mathrm{deg}, 0 \mathrm{deg})+\delta(8 \mathrm{deg}, 0 \mathrm{deg})=0 \mathrm{deg}$. The field-of-view errors of the 0 -deg contour cancel out the on-axis retardance.

similar to that of the retardation map shown in Fig. 7. Figure 8 shows how the retardance varies with the angle of incidence for achromatic and zero-order retarders.

Two points shown in Fig. 7 should be emphasized. First, the retardation $\delta(\alpha, \beta)$ is dependent only on the magnitude of the angle between the optic axis (WP1 and WP2) and the optical axis, for example, $\delta(+1 \mathrm{deg}, 0 \mathrm{deg})=\delta(-1 \mathrm{deg}, 0$ $\mathrm{deg})$ and $\delta(0 \mathrm{deg},+1 \mathrm{deg})=\delta(0 \mathrm{deg},-1 \mathrm{deg})$. Second, the retardation error becomes smaller as the angle between the optical axis and the two optic axes becomes equal, $\delta(0 \mathrm{deg}$, $0 \mathrm{deg})-\delta(\alpha, \beta) \rightarrow 0$ as $|\alpha| \rightarrow|\beta|$.

\subsection{Optic Axis Tilt Errors}

Although the optic axis tilt errors are related to the field-ofview errors, they are created when the optic axes are not perpendicular to the propagation direction of the light $(z$ axis/optical axis). Figure 9 shows four different ways errors 


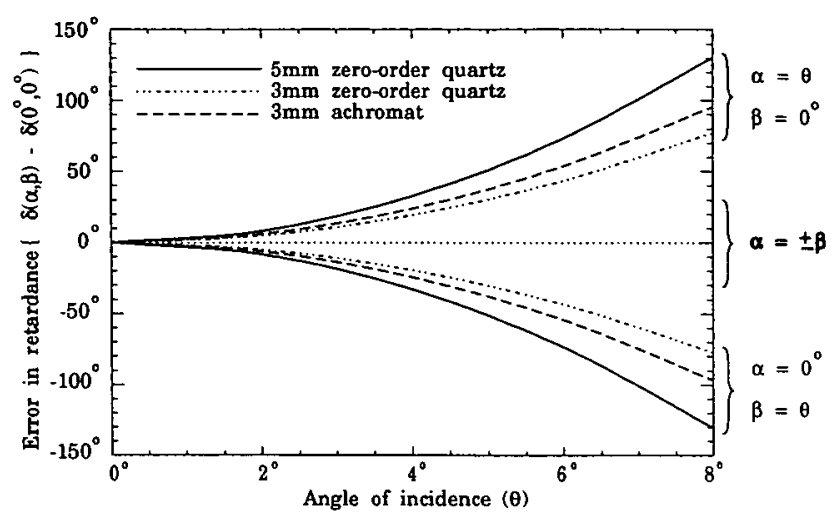

Fig. 8 Error in the retardance as a function of the incident angle $\left[\theta=\left(\alpha^{2}+\beta^{2}\right)^{1 / 2}\right]$ for both the achromatic and zero-order waveplates at $5250 \AA$. Table 1 lists the optical properties of the $5-\mathrm{mm}$ zero-order waveplate and the 3-mm achromatic waveplate.

in the alignment of the optic axes can affect the retardation measurements. In these simulations, the waveplate is mounted in a rotary stage so that measurements can be made at the four fast axis positions, 45, 135, 225, and $315 \mathrm{deg}$. This rotary stage is then allowed to tilt the waveplate with

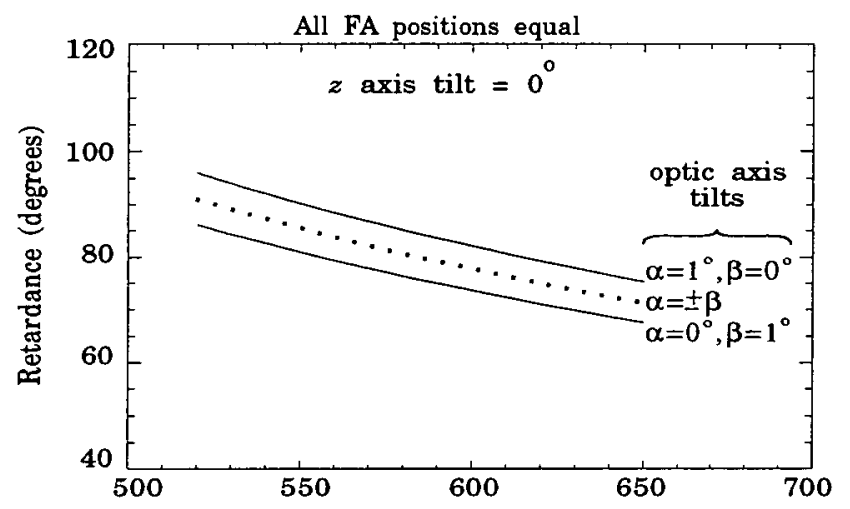

(a)

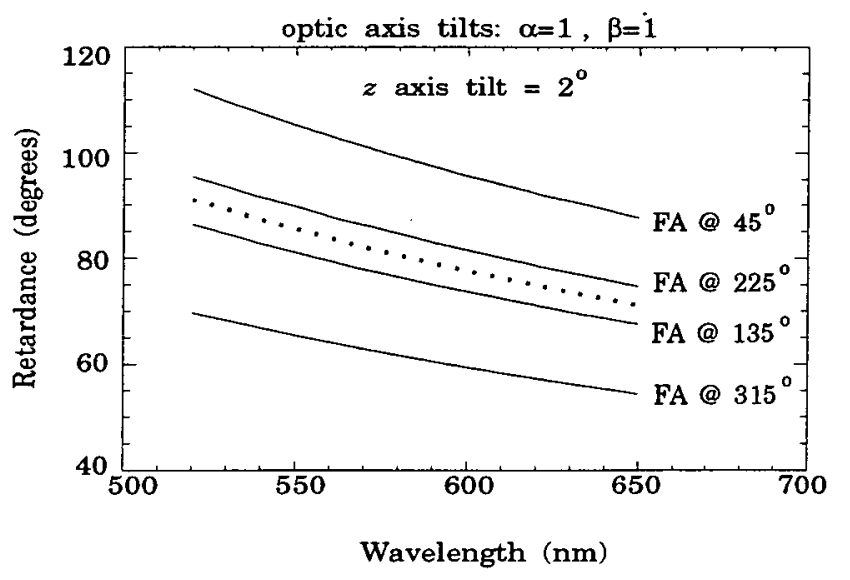

(c) respect to the $z$ axis. In these simulations, the mechanical tilt ( $z$ axis tilt in Fig. 9) was selected to maximize the retardation errors, namely, in the planes containing the WP1 or WP2 optic axes, $(\alpha, 0)$ or $(0, \beta)$ in Fig. 7.

The first optic axis error assumes that the optic axes of WP1 and WP2 are not parallel to the polished surfaces. The effect of this error is shown in Fig. 9(a). In this case, the light is assumed to be propagating along the $z$ axis, and the polished surfaces of the waveplates are perpendicular to that direction ( $z$ axis tilt $=0 \mathrm{deg}$ ). Even though the light is parallel to the $z$ axis there will be a positive error if the optic axis of WP1 is not perpendicular to the $z$ axis [Fig. 9(a), $\alpha= \pm 1 \mathrm{deg}$, $\beta=0$ deg curve], a negative retardation error if the optic axis of WP2 is tilted [Fig. 9(a), $\alpha=0 \mathrm{deg}, \beta= \pm 1 \mathrm{deg}$ curve], and no retardation error if the optic axes of WP1 and WP2 are at the same angle $(|\alpha|=|\beta|)$. When the optic axis tilt errors are equal, the two retardation errors cancel out and the errors will not be detected.

Figures $9(\mathrm{~b})$ and $9(\mathrm{c})$ demonstrate the best way to test waveplates to determine if optic axis tilt errors exist. In Fig. 9(b), it is assumed that there are no optic axis tilt errors in WP1 and WP2. The rotary stage, which holds the retarder, is tilted $2 \mathrm{deg}(z$ axis tilt $)$ with respect to the plane containing

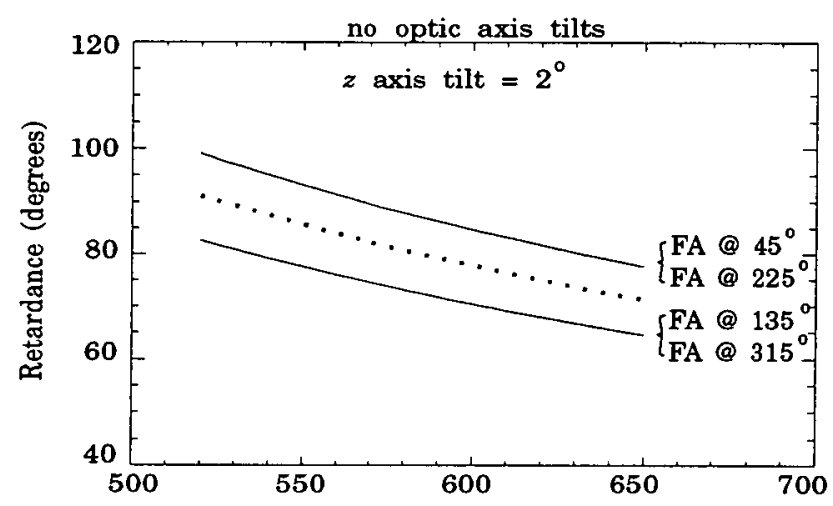

(b)

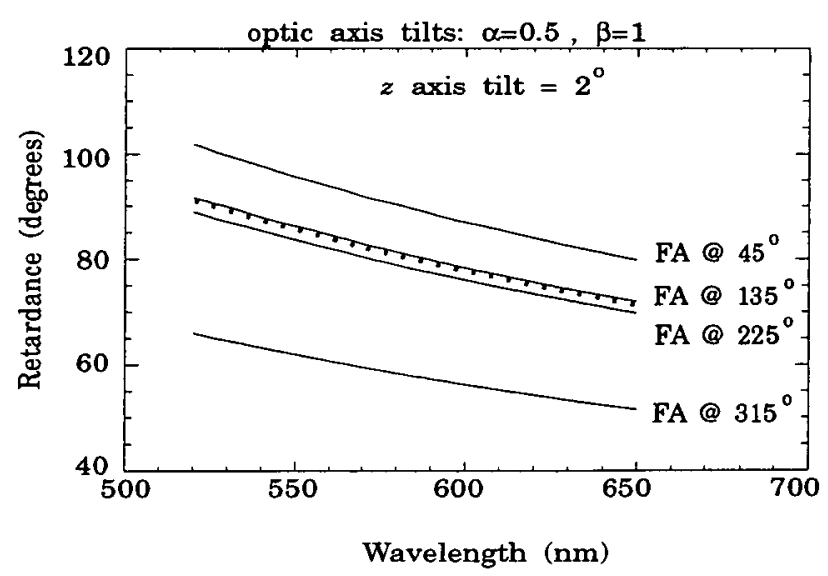

(d)

Fig. 9 Effects of optic axis errors on the retardation measurements: (a) how an optic axis tilt error will affect the measure retardation as a function of the three tilt angles ( $\alpha=1 \mathrm{deg}, \beta=0 \mathrm{deg}),(\alpha=0 \mathrm{deg}$, $\beta=1 \mathrm{deg}$ ), and $|\alpha|=|\beta|$; (b) how a 2-deg mechanical tilt of a retarder with no optic axis tilt errors will affect the retardation measurements; (c) how a mechanical tilt of 2 deg can detect the $\alpha=\beta=1$ deg optic axis tilt errors; and (d) how a 2-deg mechanical tilt will effect optic axes errors that are not equal $(\alpha=0.5$ deg, $\beta=1.0 \mathrm{deg})$. 
either the WP1 or WP2 optic axis. Now as the retarder is rotated to its fast axis locations, the optic axis $\alpha$ of WPI will be at a +2-deg tilt when the fast axis (FA) is at 45 and 225 deg. Similarly, the optic axis $\beta$ of WP2 will be at +2 -deg when the fast axis is at 135 and $315 \mathrm{deg}$. With no optic axis tilt errors, the retardation measurements at $\mathrm{FA}=45$ and 225 deg should be the same [Fig. 9(b)]. The measurements at $\mathrm{FA}=135$ and $315 \mathrm{deg}$ are also equal.

Figure 9(c) shows how internal optic axis errors affect the curves shown in Fig. 9(b). Assuming that the optic axes for WP1 and WP2 have a 1-deg tilt error, the WP1 optic axis is tilted 3 deg with respect to incident light for the 45-deg FA measurement, and at $1 \mathrm{deg}$ for the 225-deg FA measurement. This difference in the optic axis position will cause the four FA measurements to separate. Therefore, an optic axis tilt error that would go unnoticed in Fig. $9(a)(\alpha=\beta=1 \mathrm{deg})$ could be detected if the rotation axis of the retarder is tilted with respect to the propagation direction of the light. The tilt error in Fig. 9(c) is the type that should be expected in zeroorder retarders in which the birefringent material is from the same crystal. Figure 9 (d) demonstrates the effects that are expected in achromatic waveplates where two different crystals (quartz and $\mathrm{MgF}_{2}$ ) are used and the optic axis tilt errors $(\alpha, \beta)$ are not expected to be equal.

\subsection{Rotational Misalignment of the Optic Axes}

If the optic axes of a retarder are exactly $90 \mathrm{deg}$ apart, the retarder can be placed between crossed polarizers, its "fast" or "slow" axis aligned parallel to the polarizers, and there will be no detected signal. If a signal is detected, and if the birefringent crystals are not optically active, then there is a rotational misalignment [ $\rho$ in Fig. 3(d)] of the WP1 and WP2 optic axes.

The perfect zero-order quartz retarder in Table 1 is now described. With the FA of waveplate WPl at $-45 \mathrm{deg}$ to the analyzer, the retarder will transform right circular polarization $(-V)$ into elliptical polarization by creating a $-16,044$ deg phase shift $\left[\delta_{1}=-(44 \times 360 \mathrm{deg}+204 \mathrm{deg})\right]$. Because the FA of the second waveplate, WP2, is $90 \mathrm{deg}$ from the FA of WP1, it will create a positive retardation of $\delta_{2}=15,954 \mathrm{deg}$. The total retardance of WP1 and WP2 is $-90 \mathrm{deg}$, which will transform the incident circular polarization $(-V)$ into $-Q$ linear polarization. Assuming that there is an error in the alignment of the WP2 optic axis, then the circular polarization will be transformed into linear, but the linear will not be perpendicular to the analyzer (assumed to be at $+Q$ ). In effect, the retarder may be perfect $\left(\delta_{1}+\delta_{2}= \pm 90 \mathrm{deg}\right)$, but if there is a rotational misalignment of the optic axis about the optical axis ( $z$ axis), there will be linear crosstalk in the circular polarization measurement.

The main effect of this error is that the Soleil-Babinet compensator will measure two different minimum locations (or retardations) depending on whether the "fast" or "slow" axis of the retarder is aligned parallel to the input polarization. The difference in these minimum locations, which are wavelength dependent, can be used to estimate the WP2 optic axis misalignment $(\rho)$.

\section{Data Analysis}

Although Sec. 3 describes the various errors that are possible, those errors were only considered after all of the systematic and random errors in the test setup were minimized. Even though the retarders were carefully mounted in the rotary stage and observations were made of the motion of the reflected light as the retarder was rotated through $360 \mathrm{deg}$, each FA measurement $(45,225,135$, and $315 \mathrm{deg})$ had retardation values that were not equal, and repeated systematically. Only after tilting the retarders in an effort to obtain a perfect 90 -deg phase retardance did the errors in Sec. 3 become obvious.

Each retarder was measured at a minimum of four mechanical or $z$ axis tilt angles, where $\alpha_{z}$ and $\beta_{z}$ denote the orientation of the rotary stage. The mechanical tilt angle $\alpha_{z}$ is in the same plane as the WP1 optic axis tilt angle $\alpha$. Similarly, $\beta_{z}$ is in the same plane as the WP2 optic axis $\beta$. The rotary stage positions were on axis $\left(\alpha_{z}=\beta_{z}=0 \mathrm{deg}\right.$ ), one along the $\alpha_{z}$ axis $\left(\beta_{z}=0\right)$, one on the $\beta_{z}$ axis $\left(\alpha_{z}=0\right)$, and the last at $\alpha_{z}=\beta_{z}$. Figure 10 shows the measured retardance for a zero-order retarder (QWP5 in Table 2) where the rotary stage was tilted to $\left(\alpha_{z}, \beta_{z}\right)=(0.0 \mathrm{deg}, 2.3 \mathrm{deg})$. Figure 11 shows the measurements for an achromatic retarder (QWP1 in Table 2$)$ with the rotary stage tilted to $\left(\alpha_{z}, \beta_{a}\right)=(1.8 \mathrm{deg}$, $0.0 \mathrm{deg}$ ).

Although tilting the retarder $\left(\alpha_{z}, \beta_{z}\right)$ is the best way to observe the crystal optic axis errors $(\alpha, \beta)$, which were quite large in the achromatic waveplates, there is an additional uncertainty in the modeling of the achromatic retarders that was not seen in the zero-order quartz retarders. The retardation measurements made on-axis $\left(\alpha_{z}=\beta_{z}=0 \mathrm{deg}\right)$ agreed well with the computer model, but the measurements made when the achromatic retarders were tilted had a positive dc shift when compared to the computer simulations. Although a thickness error might explain this shift, the thickness is fixed by the wavelength dependence of the retarder (see Fig. 4 and Sec. 3.1). Therefore, the error is assumed to be an uncertainty in the analytical expressions used to determine the indices of refraction for the $\mathrm{MgF}_{2}$ plate. This error is listed as a birefringence error $\left(n_{x}-n_{y}\right)$ in Table 2 .

Table 2 summarizes the measurements on the retarders that were acquired for the EXVM program. Although some portion of the errors listed in Table 2 may be related to the mechanical holder that held the retarders, the "random" values of the four retarders suggest that the systematic errors in

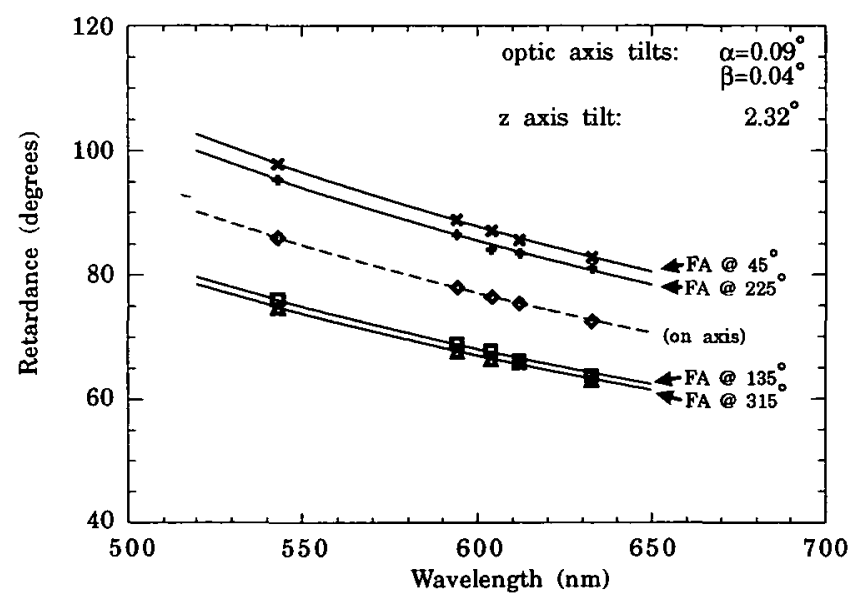

Fig. 10 Retardation measurements on a zero-order quartz waveplate (QWP5). The mechanical tilt was $(0.00 \mathrm{deg}, 2.32 \mathrm{deg})$ with internal tilt errors of $\alpha=0.09 \mathrm{deg}$ and $\beta=0.04 \mathrm{deg}$. 
Table 2 Parameters that characterize the quarter-wave plates that were tested for use in the EXVM polarimeter. The FA error of WP1 was assumed to be zero.

\begin{tabular}{|c|c|c|c|c|c|c|}
\hline \multirow[t]{2}{*}{$\begin{array}{c}\text { Retarder } \\
\#\end{array}$} & \multirow[t]{2}{*}{ Type } & \multirow[t]{2}{*}{$\begin{array}{l}\text { Retardance } \\
\text { at } 525 \mathrm{~nm} \\
\text { (degrees) }\end{array}$} & \multirow{2}{*}{$\begin{array}{c}\begin{array}{c}\text { Rotational } \\
\text { misalignment } \\
\text { (degrees) }\end{array} \\
\text { WP2 } \\
(\rho)\end{array}$} & \multicolumn{2}{|c|}{$\begin{array}{l}\text { Optic axis } \\
\text { tilt error } \\
\text { (degrees) }\end{array}$} & \multirow{2}{*}{$\begin{array}{l}\begin{array}{c}\text { Birefringence } \\
\text { error }\end{array} \\
\Delta\left(n_{x}-n_{y}\right) \\
\times 10^{6}\end{array}$} \\
\hline & & & & $\begin{array}{l}\text { WP1 } \\
(\alpha)\end{array}$ & $\begin{array}{c}\text { WP2 } \\
(\beta)\end{array}$ & \\
\hline QWP1 & achromat & $452.3 \pm 0.6$ & $0.5 \pm 0.2$ & $0.07 \pm 0.04$ & $0.95 \pm 0.05$ & $2.79 \pm 0.22$ \\
\hline QWP2 & achromat & $451.1 \pm 0.2$ & $0.5 \pm 0.1$ & $0.08 \pm 0.05$ & $1.37 \pm 0.07$ & $5.69 \pm 0.67$ \\
\hline QWP4 & zero & $91.3 \pm 0.1$ & $1.1 \pm 0.2$ & $0.07 \pm 0.04$ & $0.04 \pm 0.02$ & - \\
\hline QWP5 & zero & $88.9 \pm 0.2$ & $0.5 \pm 0.2$ & $0.07 \pm 0.02$ & $0.11 \pm 0.02$ & - \\
\hline
\end{tabular}

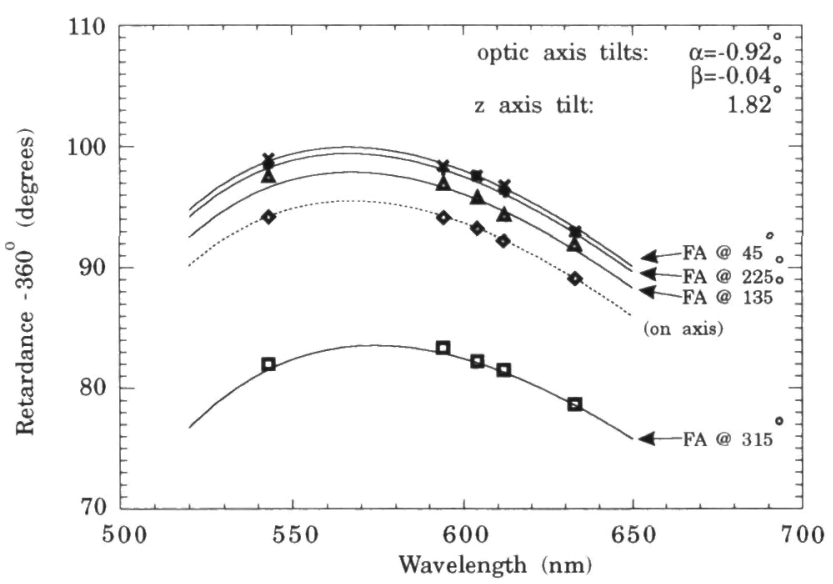

Fig. 11 Retardation measurements on an achromatic waveplate (QWP1). The mechanical tilt was (1.82 deg, $0.00 \mathrm{deg})$ with internal tilt errors in the waveplate of $\alpha=0.92 \mathrm{deg}$ and $\beta=0.04 \mathrm{deg}$.

the mechanical holder are smaller than the systematic errors in the waveplates.

\section{Conclusions}

Although knowing the average retardance (adding the four FA positions) may be acceptable in some applications, instruments that strive to measure polarization below the $10^{-3}$ level must account for the fact that the waveplate retardation may be a function of its FA (optic) position. Also, imaging instruments that are trying to measure polarization from a remote source must carefully consider the field-of-view errors when placing a waveplate in the imaging system. Although field-of-view errors in a noncollimated beam are averaged across each field position, deconvolving those errors and determining the "true"' source polarization may be difficult.
With collimated beams, each image point has a "constant" field-of-view error associated with it. This error is easy to model and can be removed from the measured polarization.

\section{References}

1. D. L. Mickey, "Vector magnetic field observations with the Haleakala polarimeter," in Measurements of Solar Vector Magnetic Fields, NASA Conference Publication 2374, M. J. Hagyard, Ed., pp. 183-191, NASA. Washington, DC (1985)

2. T. G. Baur, "Optical polarimeters for solar research," Opt. Eng. 20(1), 2-13 (1981).

3. E. A. West, "Using KD*P modulators for polarization measurements of the Sun," in Polarization Considerations for Optical Systems II, R. A. Chipman, Ed., Proc. SPIE 1166, 434-445 (1989).

4. E. A. West and M. H. Smith, "Polarization characteristics of the MSFC experimental vector magnetograph," in Polarization Analysis and Measurement II, D. H. Goldstein and D. B. Chenault, Eds., Proc. SPIE 2265, 272-281 (1994).

Edward A. West received his BS degree in engineering science from Tennessee Technological University in 1973 and his MS degree in electrical engineering from the University of Alabama in Huntsville in 1982. He joined NASA/Marshall Space Flight Center in the fall of 1973 and has been associated with the Space Science Laboratory since that time. His research interests are in the development of stable high-resolution polarimeters for both ground-based and space-based solar magnetographs and in the development of real-time analysis techniques for use in studies on solar magnetic field evolution and flare prediction.

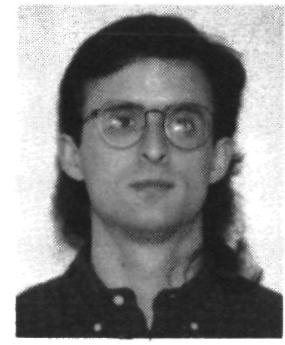

Matthew H. Smith received his BS degree in applied optics from the Rose-Hulman Institute of Technology in 1991 and his MS degree in physics from the University of Alabama in Huntsville (UAH) in 1993. He is currently pursuing his $\mathrm{PhD}$ in physics at $\mathrm{UAH}$, where his research interests include optical system design and testing of solar vector magnetographs and retinal imaging instruments. 